\title{
Carbon nanotube alignment by surface acoustic waves
}

Cite as: Appl. Phys. Lett. 85, 1427 (2004); https://doi.org/10.1063/1.1787159

Submitted: 28 April 2004 • Accepted: 29 June 2004 • Published Online: 17 August 2004

C. J. Strobl, C. Schäflein, U. Beierlein, et al.

\section{ARTICLES YOU MAY BE INTERESTED IN}

Alignment of particles in microfluidic systems using standing surface acoustic waves Applied Physics Letters 92, 044104 (2008); https://doi.org/10.1063/1.2838748

Particle concentration and mixing in microdrops driven by focused surface acoustic waves Journal of Applied Physics 104, 014910 (2008); https://doi.org/10.1063/1.2951467

Surface acoustic wave mediated dielectrophoretic alignment of rolled-up microtubes in microfluidic systems

Applied Physics Letters 96, 134105 (2010); https://doi.org/10.1063/1.3371708

母QBLOX

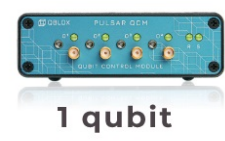

Shorten Setup Time Auto-Calibration More Qubits

Fully-integrated Quantum Control Stacks Ultrastable DC to $18.5 \mathrm{GHz}$ Synchronized $<<1$ ns Ultralow noise
View Online Export Citation

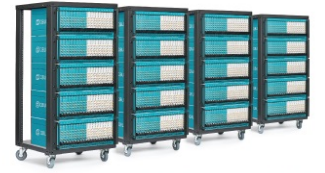

100s qubits

visit our website > 


\title{
Carbon nanotube alignment by surface acoustic waves
}

\author{
C. J. Strobl, C. Schäflein, and U. Beierlein \\ Sektion Physik der Ludwig-Maximilians-Universität and Center for NanoScience (CeNS), \\ Geschwister-Scholl-Platz 1, 80539 Munich, Germany \\ J. Ebbecke ${ }^{\text {a) }}$ and A. Wixforth \\ Institut für Physik der Universität Augsburg, Experimentalphysik I, Universitätsstr. 1, 86135 Augsburg, \\ Germany
}

(Received 28 April 2004; accepted 29 June 2004)

We present a realization of carbon nanotube alignment. Surface acoustic waves are applied to a multiwalled carbon nanotube suspension and the lateral piezoelectric field of the standing wave aligns the carbon nanotubes with an angle of $25^{\circ}$ to $45^{\circ}$ on $\mathrm{LiNbO}_{3}$ with respect to the direction of wave propagation. This angle results from a superposition of the aligning electric field and a perpendicular fluidic flux in the carbon nanotube suspension caused by the energy transfer from the surface acoustic wave into the liquid. On $\mathrm{LiTaO}_{3}$, the multiwalled carbon nanotubes align parallel to the wave vector due to negligible fluidic processes. (C) 2004 American Institute of Physics. [DOI: $10.1063 / 1.1787159$ ]

Since a report of carbon nanotube (NT) generation in $1991^{1}$ an increasing interest exists in single-walled NTs as well as in multiwalled carbon NTs (MWNTs). They are the subject of fundamental research ${ }^{2}$ as well as of direct industrial applications ${ }^{3}$ due to their remarkable mechanical, optical, and electrical properties. ${ }^{4}$

One of the limiting restrictions for a broader industrial utilization is the problem of controlled NT alignment. Selfassembly processes where the NTs are grown vertically onto a substrate material ${ }^{3,5}$ have been presented, but NT assembly or alignment parallel to the substrate surface has only be reported in a few special configurations. ${ }^{6,7}$ Here, we report an approach of surface acoustic wave (SAW)-mediated MWNT alignment parallel to the sample surface.

The MWNTs purchased were prepared at Iljin Nanotech Co. by an arc discharge method. ${ }^{8}$ The MWNTs have a diameter of $5-20 \mathrm{~nm}$ at a length of $0.5-5 \mu \mathrm{m}$. First, they were suspended in water with $1 \mathrm{wt} \%$ sodium-dodecylsulfate. The suspension was subjected to ultrasonic agitation for $10 \mathrm{~min}$ to disintegrate the MWNT network into MWNT bundles and individual MWNTs which are surrounded by surfactant shell. ${ }^{9}$ The suspension was centrifuged at $10000 \mathrm{~g}$ for 10 min to remove larger particles. One drop of NT suspension was placed in the cavity between the $\mathrm{LiNbO}_{3}$ substrate (rotation $128^{\circ}, Y$-cut, $X$ propagation) and a glass plate (see Fig. 1). Interdigital transducers (IDTs) are located on the substrate to launch a SAW with $35 \mu \mathrm{m}$ wavelength that corresponds to the resonance frequency of $114 \mathrm{MHz}$. The glass plate was located above and parallel to the substrate surface with a spacing of $20 \mu \mathrm{m}$ or less. For a time period of $30 \mathrm{~min}$, a rf signal of $30 \mathrm{dBm}$ power was applied to one IDT in order to launch a SAW with large amplitude. Finally, the sample was rinsed in deionized water and was dried with nitrogen gas.

In Fig. 2, atomic force microscope (AFM) images (in tapping mode) are shown of four $\mathrm{LiNbO}_{3}$ samples with different MWNT concentrations. A preferential direction of NT alignment between $\pm 25^{\circ}$ and $\pm 45^{\circ}$ [with respect to the direc-

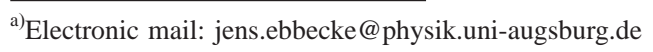

tion of SAW propagation; indicated in Fig. 2(a)] is identifiable. The sign of the angle was not dependent on the MWNT concentration. In Fig. 2(d), a symmetric alignment in positive and negative direction is visible (at higher NT concentrations) that leeds to a crossing of the MWNT with an angle from $50^{\circ}$ to $90^{\circ}$. These three configurations were observed throughout the whole acoustic path of the sample, i.e., the region that was treated by the SAW. By varying the NT concentration in the suspension, the average separation length can be varied efficiently (see Fig. 3). With increasing MWNT concentration, the average separation distance of the individual MWNTs decreases. The distribution width is about $30 \%$ of the average separation value.

Several experiments were carried out to understand factors controlling the MWNT alignment. In order to understand the role of the SAW-induced electric field in the alignment procedure, one sample was covered with a thin layer of $\mathrm{NiCr}(20 \mathrm{~nm})$ before the suspension was applied. The piezoelectric field of the travelling wave is then screened by the metallic film at the surface of the substrate and no alignment was observed with this sample (Fig. 4).

The SAW launched on the $\mathrm{LiNbO}_{3}$ samples presented here is a Rayleigh wave and the crystal particle movement has longitudinal and transversal components. The transversal component is responsible for the good fluidic functionality of this material. On $\mathrm{LiTaO}_{3}$ (rotation $36^{\circ}, Y$-cut, $X$ propagation) a shear-wave SAW (SH-SAW) can be launched with IDTs rather than a Rayleigh wave. A SH-SAW has only a mechanical component in the plane of the crystal and is therefore less fluidic active than a Rayleigh wave. In Fig. 5, an excellent
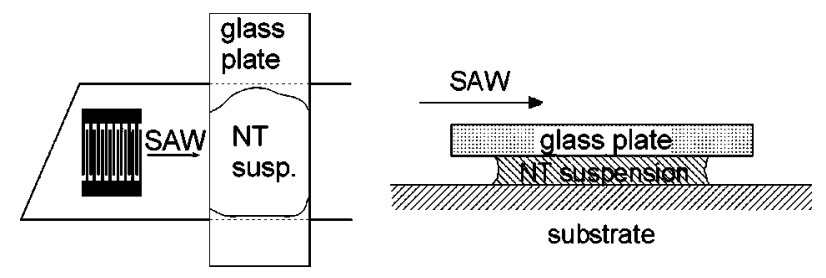

FIG. 1. Experimental setup: One drop of NT suspension is placed in the cavity between the $\mathrm{LiNbO}_{3}$ substrate and a glass plate. 

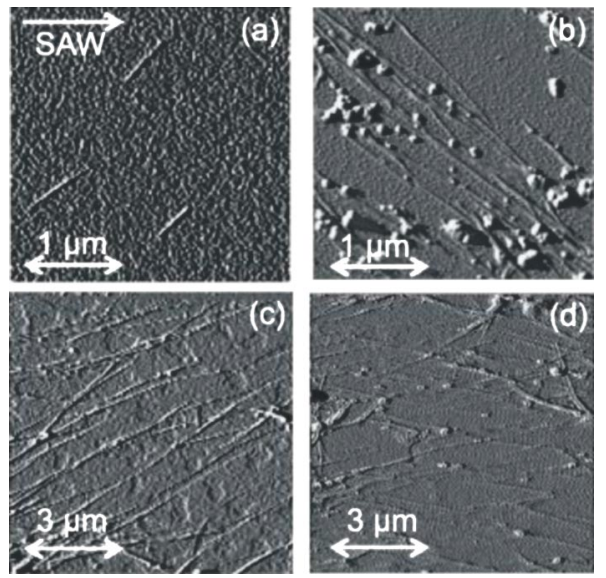

FIG. 2. AFM images of aligned MWNTs with different concentrations of the NT suspension. After treatment with the SAW on $\mathrm{LiNbO}_{3}$, the MWNTs are aligned with an angle between $\pm 25^{\circ}$ and $\pm 45^{\circ}$.

MWNT alignment on $\mathrm{LiTaO}_{3}$ as substrate material is presented. The sample was processed in the same way with MWNTs and SAW treatment as described above. The SHSAW propagates at the substrate surface and is accomplished by an ac electric field that leaks into the NT suspension. As a result of these investigations, we can deduce that the parallel component of the piezoelectric field is the driving force of MWNT alignment and the mechanical and fluidic processes have less of a pronounced effect.

In the following, we discuss the question why the MWNTs on $\mathrm{LiNbO}_{3}$ align with an angle of $\pm 25^{\circ}$ to $\pm 45^{\circ}$. In order to investigate this effect, we carried out the same experiments on $\mathrm{LiNbO}_{3}$ as shown in Fig. 1 with latex spheres instead of MWNTs (see Fig. 6). The spheres had an average diameter of $5 \mu \mathrm{m}$ and were big enough to be observed by a light microscope coupled to a charge coupled device camera during the experiment. We were able to vary the spacing between the glass plate and the surface of the substrate and recorded the alignment of the spheres at the same time. If the spacing was of the order of one SAW wavelength or less, a standing water pressure wave arose and the spheres aligned along stripes parallel to the wave front of the standing wave. The spacing between the lines of latex spheres was $21 \mu \mathrm{m}$ (small period in Fig. 6) that is one-half of the wavelength of the water pressure wave was induced by the SAW. This water pressure wave evolves from the energy transfer from the SAW to the liquid. Strong fluidic processes in the suspension were observed with a pronounced component perpendicular to the wave vector of the SAW. But in addition to this fluidic flux, a standing wave with a period in

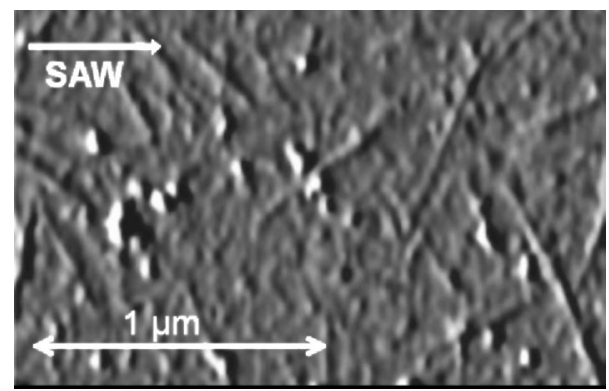

FIG. 4. AFM picture of a metallized $\mathrm{LiNbO}_{3}$ sample: No alignment of the MWNTs.

the order of $150 \mu \mathrm{m}$ could be seen at a particular spacing between the glass plate and the surface of the substrate (the hyperperiod in Fig. 6). Furthermore, it is worth mentioning that an identical dependence of the MWNT alignment on the existence of standing water pressure waves was experimentally detected on $\mathrm{LiNbO}_{3}$.

The combination of the alignment by the electric field and the fluidic effect seems to be the reason for the NT alignment with an angle of $25^{\circ}$ to $45^{\circ}$ on $\mathrm{LiNbO}_{3}$. The polarizable MWNTs try to align along the electric-field vector that has acomponent perpendicular and a component parallel to the sample surface. MWNTs close to the sample surface (and only there) attach to the surface at one end, and the fluidic flux forces the MWNT to rotate clockwise (or counterclockwise depending on the direction of the flux and which end of the NT attaches first to the surface) until equilibrium is reached. Finally, the MWNTs settle down with an angle between $25^{\circ}$ and $45^{\circ}$.

We believe that our approach of NT alignment to produce parallel arrays or crossed structures offers advantages over current efforts like random deposition, ${ }^{10}$ and direct manipulation of individual nanowires and $\mathrm{NTs},{ }^{11}$ and electric field. ${ }^{12-15}$ With random deposition or direct manipulation, it is laborious and time consuming to obtain parallel arrays or crossed structures for integrated nanodevices. Assembling with the help of electric fields offers a higher throughput but needs extensive lithography to fabricate the electrodes required for the assembly of NTs or nanowires device structures. These studies provide a general approach for NT alignment into parallel arrays with control of the average separation. Therefore, SAW-mediated NT alignment represents a general strategy for the organization of nanowires and NTs building blocks into structures needed for wiring, interconnections, and functional devices, and thus could enable a bottom-up manufacturing process for future nanotechnologies.

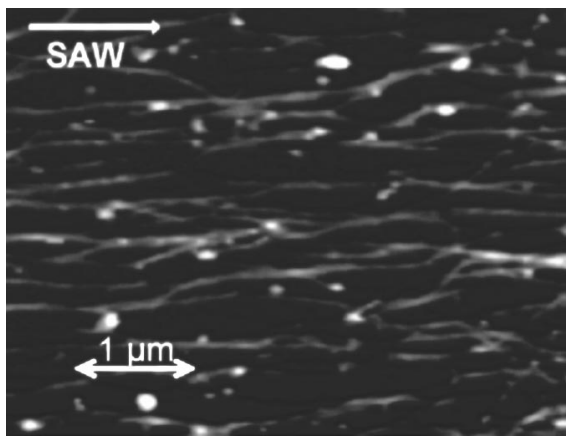

FIG. 5. AFM picture of aligned MWNTs on $\mathrm{LiTaO}_{3}$ as substrate material. 


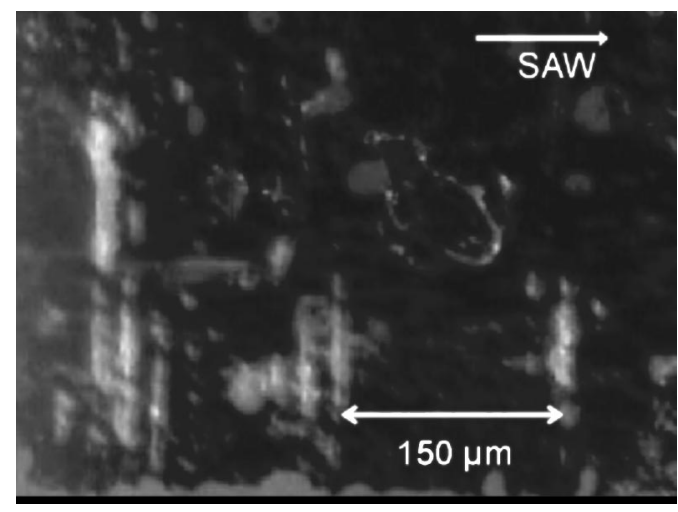

FIG. 6. Latex spheres with an average diameter of $5 \mu \mathrm{m}$ align along lines perpendicular to the wave vector of the SAW.

The authors like to thank the Advalytix AG for their support. This work was funded by the Bayrische Forschungsstiftung (FORNANO).

${ }^{1}$ S. Iijima, Nature (London) 354, 56 (1991).
${ }^{2}$ R. Egger, Phys. Rev. Lett. 83, 5547 (1999).

${ }^{3}$ W. B. Choi, D. S. Chung, J. H. Kang, H. Y. Kim, Y. W. Jin, I. T. Han, Y. H. Lee, J. E. Jung, N. S. Lee, G. S. Park, et al., Appl. Phys. Lett. 75, 3129 (1999).

${ }^{4}$ T. W. Ebbesen, Phys. Today, 26 (1996).

${ }^{5}$ Z. P. Huang, J. W. Xu, Z. F. Ren, J. H. Wang, M. P. Siegal, and P. N. Provencio, Appl. Phys. Lett. 73, 3845 (1998).

${ }^{6}$ M. Burghard, G. Duesberg, G. Philipp, J. Muster, and S. Roth, Adv. Mater. (Weinheim, Ger.) 10, 584 (1998).

${ }^{7}$ J. Liu, M. J. Casavant, M. Cox, D. A. Walter, P. Boul, W. Lu, A. J. Rimberg, K. A. Smith, D. T. Colbert, and R. E. Smalley, Chem. Phys. Lett. 303, 125 (1999).

${ }^{8}$ T. W. Ebbesen and P. M. Ajayan, Nature (London) 358, 220 (1992).

${ }^{9}$ G. S. Duesberg, M. Burghard, J. Muster, G. Philipp, and S. Roth, Chem. Commun. (Cambridge) 1998, 435 (1998).

${ }^{10}$ M. S. Fuhrer, J. Nygard, L. Shih, Y.-G. Yoon, M. S. C. Mazzoni, H. J. Choi, J. Ihm, S. G. Louie, A. Zettl, and P. L. McEuen, Science 288, 494 (2000).

${ }^{11}$ T. Rueckes, K. Kim, E. Joselevich, G. Y. Tseng, C.-L. Cheung, and C. M. Lieber, Science 289, 94 (2000).

${ }^{12}$ A. K. Boal, F. Ilhan, J. E. DeRouchey, T. Thurn-Albrecht, T. P. Russell, and V. M. Rotello, Nature (London) 404, 746 (2000).

${ }^{13}$ R. C. Hayward, D. A. Sayille, and I. A. Aksay, Nature (London) 404, 56 (2000).

${ }^{14}$ M. Li, H. Schnablegger, and S. Mann, Nature (London) 402, 393 (1999).

${ }^{15}$ K. Yamamoto, S. Akita, and Y. Nakayama, J. Phys. D 8, 34 (1998). 